\title{
Acute Malnutrition among Children at Time of Cancer Diagnosis at a Paediatric Oncology Centre in South West Uganda
}

\author{
Ndayisenga jeanine ${ }^{1}$, Atwiine Barnabas ${ }^{2}$, Francis Oriokot ${ }^{2}$, Atwiine Daniel ${ }^{2}$, Stella \\ Kyoyagara $^{2}$, and Kevin Schwartz ${ }^{3}$ \\ ${ }^{1}$ Mbarara University of Science and Technology Faculty of Science \\ ${ }^{2}$ Mbarara University of Science and Technology Faculty of Medicine \\ ${ }^{3}$ Massachusetts General Hospital
}

March 5, 2021

\begin{abstract}
Background: Acute malnutrition is a frequent comorbidity in children with cancer and is associated with poor cancer treatment outcomes. This study describes the prevalence of acute malnutrition and its predictors at time of cancer diagnosis at Mbarara Regional Referral Hospital (MRRH). Methods: This was a retrospective chart review of children <15 years of age at diagnosis of cancer seen at the Children's Cancer Unit of MRRH between May 2017 and May 2019. Using a data extraction tool, information regarding demographics, anthropometry at admission and cancer diagnosis was extracted from the children's medical records. Descriptive statistics and logistic regression analysis were used to determine the prevalence and patterns of acute malnutrition among children at diagnosis of cancer and the factors associated with acute malnutrition, respectively. The study was approved by the Mbarara University of Science and Technology Research and Ethics Committee. Results: One hundred and thirty-three children were included in the study with a mean age of 6.6 years (SD 3.9).54.9\% of the children were male. Overall, 46 (34.6\%) of subjects had acute malnutrition at time of cancer diagnosis. Of these, $25(54.3 \%)$ had moderate acute malnutrition and $21 / 46(45.7 \%)$ had severe acute malnutrition. The factors independently associated with acute malnutrition after multivariate logistical regression analysis were age $>5$ years $(\mathrm{p}<0.0001)$ and reduced appetite $(\mathrm{p}=0.002)$. Conclusion: Acute malnutrition is common among children with cancer at diagnosis, especially in older children and those reporting poor appetite. Nutrition assessment and care should be incorporated into routine childhood cancer care to improve cancer treatment outcomes.
\end{abstract}

\section{Hosted file}

Jeanine Ndayisenga manusript on acute malnutrition in childhood cancer in Uganda doc.pdf available at https://authorea.com/users/399774/articles/512204-acute-malnutrition-amongchildren-at-time-of-cancer-diagnosis-at-a-paediatric-oncology-centre-in-south-westuganda 